\title{
Complex character Analysis of Shenzhen 100 index Stock Market Network
}

\author{
Yang Tu ${ }^{a}$, Zhihui Yang ${ }^{b^{*}}$ \\ School of Statistics and Applied Mathematics, Anhui University of Finance and Economics, Bengbu \\ 233030, China \\ a email:18895689136@163.com, bemail: zhi-hui-yang@163.com \\ * the corresponding author
}

Keywords: Stock market network; Correlation coefficient; Small world; Centrality analysis

Abstract. By the data of these stocks, daily closing prizing, volume, and return, we built three kinds of stock market network which based on the concept of complex network. First of all, we calculated the correlation index between stock nodes, which were expressed by a correlation coefficient matrix. According to kernel density distribution of daily closing prizing, volume, and return s correlation coefficient ,we determined their threshold and established the stock correlation networks. Then we analyzed character of small world for these stock correlation networks. Finally, by indicator for degree and betweenness centrality, we analyzed centrality for trading volume stock market network, which have strongest character of small world comparing with other networks.

\section{Introduction}

The study of complex networks gradually rose in the 1990s, attracting a large number of domestic and foreign research scholars. Many scholars could apply complex network theory to various fields, such as: enterprise network, the Internet, income distribution networks, knowledge dissemination network, telephone network, stocks networks. Matthew K. Meisel [1] applied egocentric social network analysis (SNA) to investigate the prevalence of addictive behavior and co-occurring substance use in college students' networks. The result showed that individuals co-occurring addictive behavior is spread evenly throughout networks. Sandra Molano [2] use the analysis of the social networks among CENDES COLOMBIA beneficiaries, and its incidence in the intervention process. Then, Key features of the CENDES beneficiaries, such as connection strength, degree of separation, and degree of centrality are discussed, showing as results that people associated to this process is connected with a low density in the entire network created, but with a greater tendency to clustering, reflecting teamwork.

Nowadays, stock market play important roles in national economy, because it implicated circulation market value of GDP. Meanwhile, stock market s research can help investors know the structure and characteristics of the stock market and analyze the operation law of the stock market . To a certain extent, it can reduce investment risk. Therefore, many scholars study the theory of complex networks for the stock market. According to given threshold, Tse Chi K [3] established stock correlation network with winner-win standard. Mansooreh Kazemilari [4] introduced the concept of vector relation, and assumed each stock is a multivariate time series. Then, he established stock correlation network, analyzed the structure of minimum dendrogram. In addition, some scholars analyzed specific characteristic of stock network. Lee [5] selected 200 stocks in Korea KOSPI and established their stock network. Then, he analyzed average path length and degree distribution in the stock network. In recent years, domestic research of stock network is increasing. Zhihui Yang [6] selected 284 stocks which contains 13 sectors in 300 stock in Shanghai and Shenzhen stock market ,and established stock market network by these stock's daily return. Then he analyzed the character of the stock network, and the result showed that the stock market had character of small world. In this paper, we absorbed some advantages from above analysis of stock network. First of all, we established stock market network by the data of these stocks , daily closing prizing, volume, and 
daily return. Then we analyzed centrality of these network. Finally, we tested whether the stock daily return have character of small world.

\section{Construction of network model}

Date selection. In this paper, we selected 87 stocks in Shenzhen 100 index as the research object. Basing on statistical indicators of their closing price, trading volume, daily return rates, we established stock market network. The time of sample data is from January 6, 2015 to April 3, 2015.

Correlation coefficient matrix of stock network. In the stock correlation network, the correlation coefficients $\rho_{i j}$ between $j$ and $i$ can be expressed as follows:

$$
\rho_{i j}=\frac{E\left(v_{i} v_{j}\right)-E\left(v_{i}\right) E\left(v_{j}\right)}{\sqrt{\operatorname{Var}\left(v_{i}\right)} \sqrt{\operatorname{Var}\left(v_{j}\right)}}, \operatorname{Var}\left(v_{j}\right)=\frac{1}{T-2} \sum_{t=1}^{T-1}\left(v_{j}(t)-E\left(v_{j}\right)\right)^{2}, \operatorname{Var}\left(v_{i}\right)=\frac{1}{T-2} \sum_{t=1}^{T-1}\left(v_{i}(t)-E\left(v_{i}\right)\right)^{2}
$$

where $v_{i}$ and $v_{j}$ respectively represents a series of stocks $i, j$ related statistical indicators .E( $\left.v_{i}\right)$ and $E\left(v_{j}\right)$ represent the average number of stock $i, j$ related statistical indicators in the period of $\mathrm{T}-1 . E\left(v_{i} v_{j}\right)$ represent the average number of related statistical indicators multiplying between stock $i$ and stock $j$ in the period of T- 1 .

In the next, we established stock market networks, which respectively based on the indicators of closing prizing, volume, and return. So at first of all, we respectively calculated correlation matrix of above indicators. Let $p_{i}, p_{j}$ indicating respectively closing price series of stock $i, j$, which substituted $v_{i}, v_{j}$ in Eq. 1. In this way, we can obtain correlation matrix of closing prizing (part of matrix was shown in Table 1 . Similarly, by Eq. 1 , let $q_{i}, q_{j}$ indicate respectively trading volume series of stock $i, j$ (Part of matrix was shown in Table 2), and let $r_{i}, r_{j}$ indicated respectively daily return series of stock $i, j$ (part of matrix was shown in Table 3).

Table 1. Correlation matrix of closing price

\begin{tabular}{|c|c|c|c|c|c|}
\hline & 1 & 2 & 3 & 4 & 5 \\
\hline 1 & 0 & 0.623 & 0.611 & 0.532 & 0.68 \\
\hline 2 & 0.623 & 0 & 0.587 & 0.468 & 0.674 \\
\hline 3 & 0.611 & 0.587 & 0 & 0.95 & 0.895 \\
\hline 4 & 0.532 & 0.468 & 0.95 & 0 & 0.88 \\
\hline 5 & 0.68 & 0.674 & 0.895 & 0.88 & 0 \\
\hline
\end{tabular}

Table 2. Correlation matrix of trading volume

\begin{tabular}{|c|c|c|c|c|c|}
\hline & 1 & 2 & 3 & 4 & 5 \\
\hline 1 & 0 & 0.51 & 0.36 & 0.076 & 0.314 \\
\hline 2 & 0.51 & 0 & 0.401 & 0.155 & 0.379 \\
\hline 3 & 0.36 & 0.401 & 0 & 0.518 & 0.641 \\
\hline 4 & 0.076 & 0.155 & 0.518 & 0 & 0.407 \\
\hline 5 & 0.314 & 0.379 & 0.641 & 0.407 & 0 \\
\hline
\end{tabular}

Table 3. Correlation matrix of daily return

\begin{tabular}{|c|c|c|c|c|c|}
\hline & 1 & 2 & 3 & 4 & 5 \\
\hline 1 & 0 & 0.7 & 0.402 & 0.426 & 0.534 \\
\hline 2 & 0.7 & 0 & 0.362 & 0.351 & 0.366 \\
\hline 3 & 0.402 & 0.362 & 0 & 0.524 & 0.523 \\
\hline 4 & 0.426 & 0.351 & 0.524 & 0 & 0.529 \\
\hline 5 & 0.534 & 0.366 & 0.523 & 0.529 & 0 \\
\hline
\end{tabular}

Determination of threshold. Using $\rho_{i j}$ representing correlation coefficient between stock $i, j$, if $\left|\rho_{i j}\right|>\theta(\theta \in[-1,1])$, we believed that there is a edge between stock $i$ and stock $j$, and defined $\theta$ as threshold. To determine threshold, we respectively should draw kernel density distribution of closing 
price, trading volume, daily return correlation coefficient. As is shown in Fig. 1, we concluded that threshold of stock closing price network equal to 0.9 , because most of closing price correlation coefficient distributed in interval of [0.7, 0.9], especially in point of 0.9 . If the correlation coefficient was greater than 0.9 , we believed that there is a edge between nodes, otherwise there is no edge between nodes. In the same way, according to kernel density distribution of trading volume, we concluded that its threshold equal to 0.4 . Similarly,according to kernel density distribution of daily return, we concluded that its' threshold equal to 0.3 .

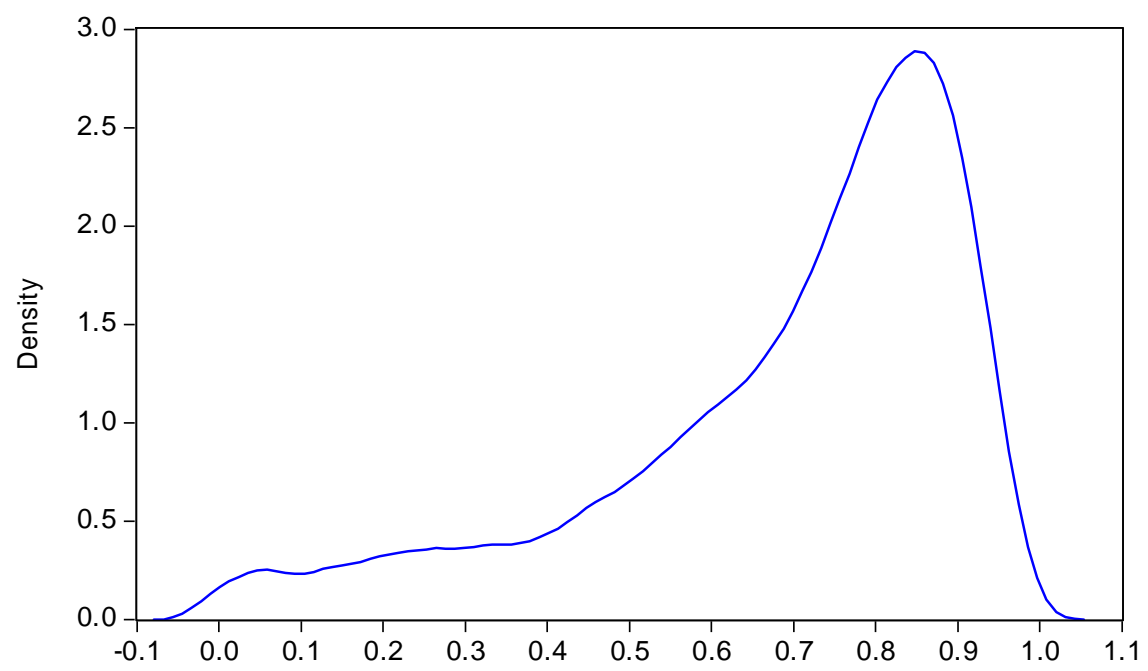

Figure 1. Kernel density distribution of stock closing price

Construction of network. According to correlation coefficient matrix of closing price, trading volume, daily return and relative threshold, which was calculated previously, we can respectively construct stock market network of closing price, trading volume, daily return. These networks were respectively shown in Fig. 2, Fig. 3 and Fig. 4.

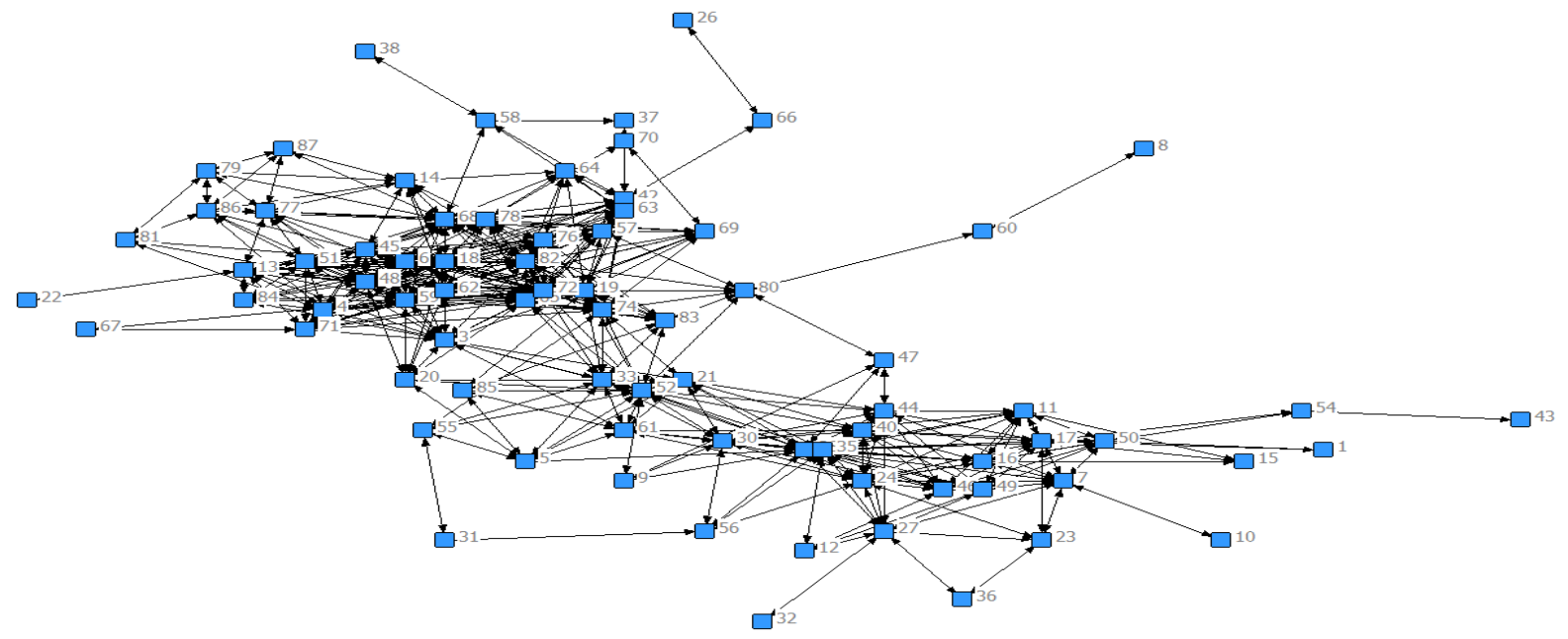

Figure 2. Network of stock closing price according to $\theta=0.9$ 


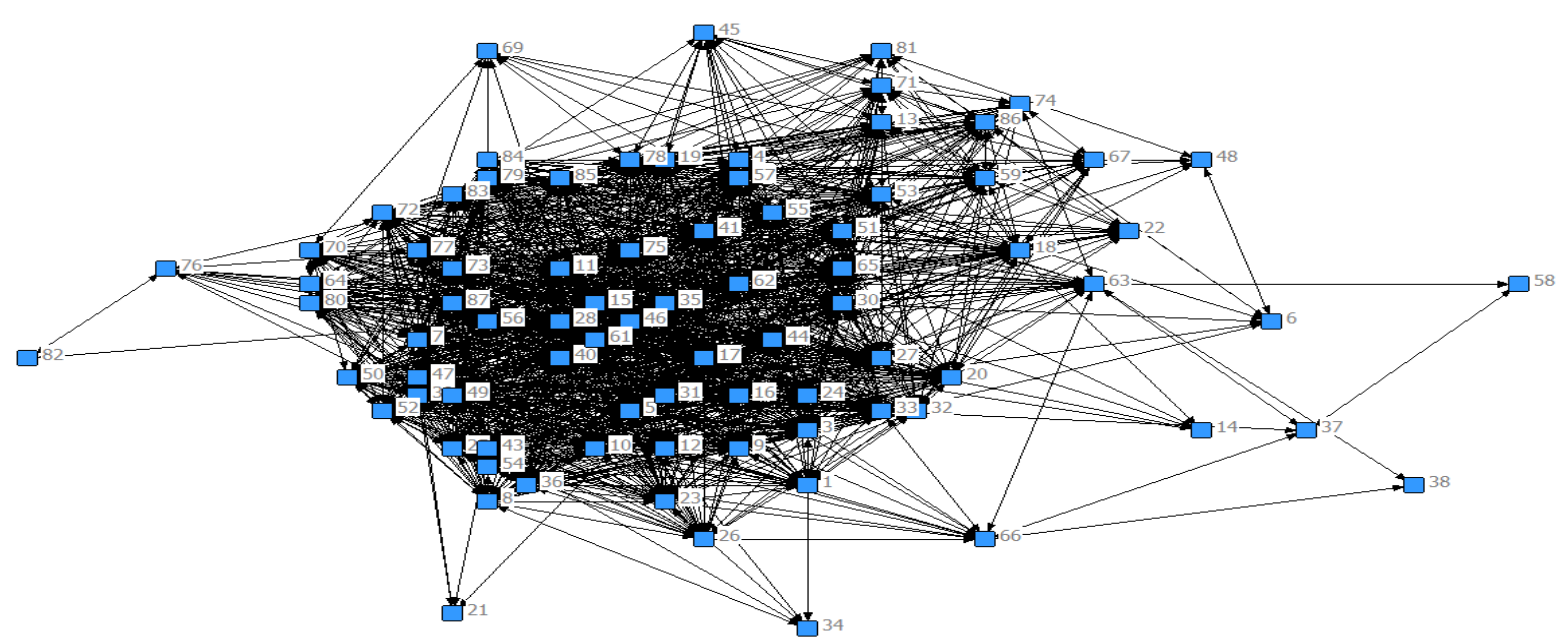

Figure 3. Network of stock trading volume according to $\theta=0.4$

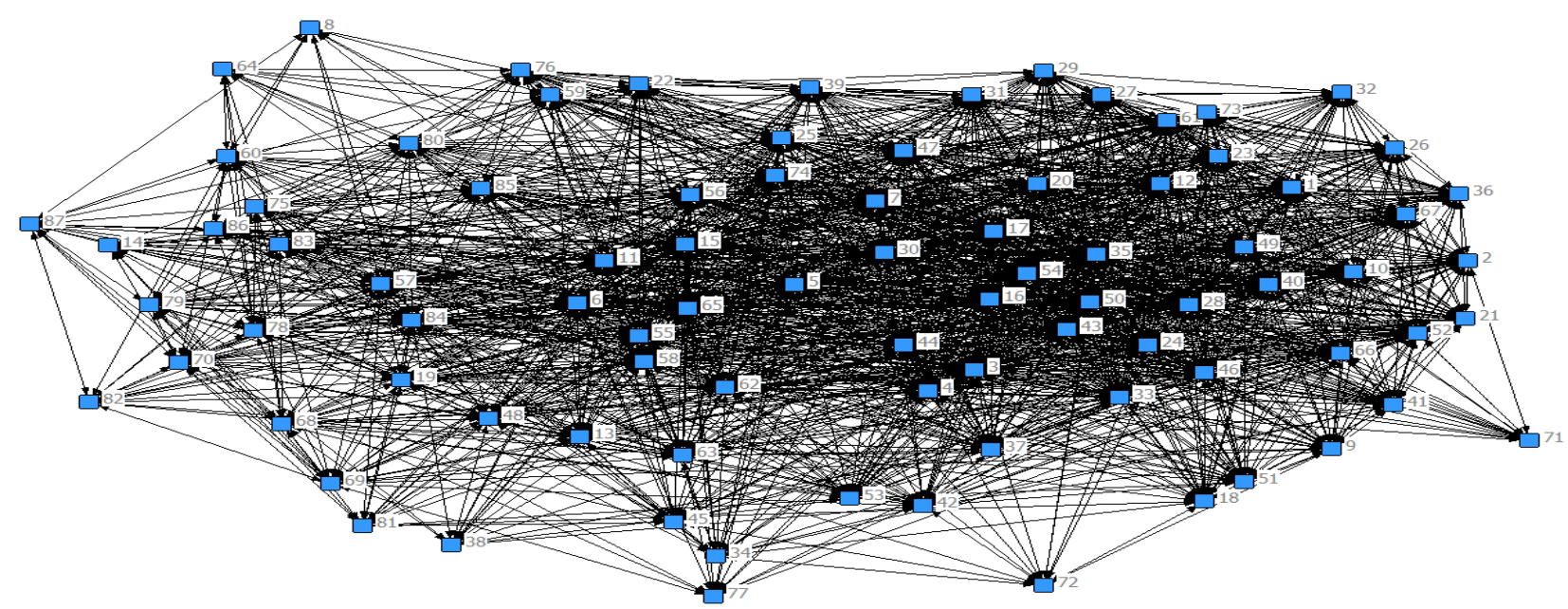

Figure 4. Network of stock daily return according to $\theta=0.3$

\section{The Small World test of stock market network}

Small World refer to the future of a huge network, in which we can establish contract by a few steps between any node. We can test Small World of network by measures of the feature length and clustering coefficient.

Feature length. Feature length indicates the average length of any two nodes' shortest route in network, usually referred to as $\mathrm{L}$, that is $L=2 \sum_{i>j} l_{i j} / n(n-1)$. where $n$ represents the total number of nodes in the network, $l_{i j}$ represents the shortest way in node $i$ and node $j$.

Clustering coefficient. In an directionless and weightless network $G=(N, g)$, we could sign clustering coefficient of node $i$ as $C_{i}$, which are expressed as $C_{i}=k_{i} / h_{i}$. Where $k_{i}$ is equal to the number of closed triads, which contains node $i$, and $h_{i}$ is equal to the number of triads, which also contains node $i$. We could sign clustering coefficient of the whole network as $C$, which is shown as $C=\left(\sum_{i} C_{i}\right) / n$. we respectively tested Small World characteristic of closing price, trading volume, daily stock network by UCINET. The Result was shown in Table 4. 
Table. 4. The measure of small world indicators

\begin{tabular}{ccc}
\hline & feature length & $\begin{array}{c}\text { clustering } \\
\text { coefficient }\end{array}$ \\
\hline Correlation network of stock closing price & 2.988 & 0.604 \\
Correlation network of stock trading volume & 1.562 & 0.744 \\
Correlation network of stock daily return & 1.497 & 0.696 \\
\hline
\end{tabular}

By Table.4, we can make the following conclusion: The feature length of stock trading volume network and stock daily return network are respectively $1.562,1.497$. Their feature lengths are both between 1-2, which indicated that any two nodes were established connection in the these two network only by one or two steps. Therefore, the relevance between nodes is strong in stock trading volume network and stock daily return network. On the contrary, the feature length of stock closing price network is 2.998. Its' feature length is closed to 3, which indicated that any two nodes were established connection in the network by three steps. Therefore, the relevance between nodes is weak in stock closing price network. The clustering coefficient of stock trading volume network and stock daily return network are respectively $0.696,0.744$, and these two clustering coefficient are high. However, the clustering coefficient of stock closing price network is 0.604 , and clustering coefficient is not high. To sum up, stock trading volume network and stock daily return network have character of small world because of their long feature lengths and high clustering coefficient, however, stock closing price network does not have character of small world, because of their short feature lengths and low clustering coefficient.

\section{Centrality of stock network}

Centrality of network discuss how much right individuals or organizations have much in social networks. In this paper, by indicator for degree and betweenness centrality, we analyzed centrality for trading volume stock market network, which have strongest character of small world comparing with other two networks.

Degree centrality. degree centrality of a node shows how many other nodes that this node can directly link with. Generally, we use the indicator of absolute or relative degree centrality to express. The absolute degree centrality of node $i$ can be expressed as $C_{A D}(i)=d_{i}=\sum_{j \in G} g_{i j}$. There is a link between node $i$ and node $j$ if $g_{i j}=1$, otherwise $g_{i j}=0$. The relative degree centrality of node $i$ can be expressed as $C_{R D}(i)=C_{A D}(i) /(\mathrm{n}-1)$. we analyzed degree centrality in stock trading volume network, part of the result was shown in Table 5.

Table 5. The result of degree centrality in stock network

\begin{tabular}{cccccc}
\hline node & name of stock & code & $\begin{array}{c}\text { Absolute } \\
\text { degree } \\
\text { centrality }\end{array}$ & $\begin{array}{c}\text { relative degree } \\
\text { centrality }\end{array}$ & rank \\
5 & ZhongXingTongXun & 000063 & 55 & 0.639 & 1 \\
54 & XiShanMeiDian & 000983 & 54 & 0.627 & 2 \\
17 & JinYuanMeiDian & 000552 & 52 & 0.604 & 3 \\
50 & JiZhongNengYuan & 000937 & 52 & 0.604 & 3 \\
16 & GuangDongDianLiA & 000539 & 51 & 0.593 & 5 \\
\hline
\end{tabular}

By Table.5, the degree centrality of ZhongXingTongXun is highest in the stock network. Its' degree centrality is 55, meaning that when its' trading volume changes, it can directly affect the turnover of 55 stocks. At the same time, trading volume of ZhongXingTongXun stock was influenced by these 55 stocks.

Betweenness centrality. Betweenness centrality measure that how important one node plays mediating role in network, which can test the node's ability of controlling resource in the network. We can measure one node's betweenness centrality by two indicators, namely absolute betweenness centrality and relative betweenness. The absolute betweenness centrality of node $i$ can be expressed 
as $C_{A B i}=\sum_{j} \sum_{k} b_{j k}(i), j \neq k \neq i, j<k$, and the relative betweenness centrality of node $i$ can be expressed as $C_{R B i}=2 C_{A B i} /\left(n^{2}-3 n+2\right)$, where $b_{j k}(i)$ indicate the possible that $i$ is located in shortest way between $i$ and $j$. We analyzed the betweenness centrality for stock trading volume network, part of the result was shown in Table 6:

Table 6. The result of betweenness centrality in stock network

\begin{tabular}{cccccc}
\hline node & name of stock & code & $\begin{array}{c}\text { Absolute } \\
\text { degree } \\
\text { centrality }\end{array}$ & $\begin{array}{c}\text { relative } \\
\text { degree } \\
\text { centrality }\end{array}$ & rank \\
\hline 11 & DongEEJiao & 000423 & 262.824 & 0.07197 & 1 \\
5 & ZhongXingTongXun & 000063 & 246.811 & 0.06753 & 2 \\
7 & WeiChaiDongLi & 000338 & 166.360 & 0.04552 & 3 \\
56 & XinHeCheng & 002001 & 164.070 & 0.04489 & 4 \\
65 & LaiBaoGaoKe & 002106 & 134.880 & 0.03690 & 5 \\
\hline
\end{tabular}

By Table 6, the betweenness centrality of DongEEJiao is highest in the stock network. Its' betweenness centrality is 286.824 , indicating that it play much mediating role in network. So DongEEJiao is located in core position in the whole stock network.

\section{Conclusion}

From above analysis, we can make following conclusions: Stock trading volume network and stock daily return network have character of small world. However, stock closing price network does not have character of small world. In the stock trading volume network, the degree centrality of ZhongXingTongXun is highest, so it link with other stocks most closely. the betweenness centrality of DongEEJiao is highest, so it has strongest ability of controlling resource.

Moreover, the establishment of the Shenzhen 100 Index stocks networks and the structural analysis for network reflected the changes of the stock market, provided a theoretical basis for analyzing the interaction between the stocks, and also provided theoretical consult for stock investment. At the same time, this study reflected to some extent situation of the current national economic circulation, which helping government to make relevant economic policies. It is greatly significant and value for stability and harmonious of national economy.

\section{References}

[1] Meisel M K, Clifton A D, MacKillop J, et al. A social network analysis approach to alcohol use and co-occurring addictive behavior in young adults, Addictive Behaviors. 51 (2015) 72-79.

[2] Molano S, Polo A. Social Network Analysis in a Learning Community. Procedia Social and Behavioral Sciences, 185 (2015) 339-345.

[3] Tse Chi K, Liu J, Lau F C M. A network perspective of the stock market. Journal of Empirical Finance, 17 (4) (2010) 659-667.

[4] Kazemilari M, Djauhari M A. Correlation network analysis for multi-dimensional data in stocks market, Physica A: Statistical Mechanics and its Applications. 429 (2015) 62-75.

[5] Lee K E, Lee J W, Hong B H. Complex networks in a stock market, Computer physics communications. 177 (2007) 186.

[6] Zhihui Yang, Hanmei Jia. Network structure analysis of stock returns correlation,Chinese Association of Automation Volume B. Beijing: Science Press, (2011) 5732-5736. "In Chinese" 